УДК 681.5

\title{
СИСТЕМА УПРАВЛЕНИЯ УСТРОЙСТВОМ КОНТРОЛЯ ПРИТОКА ФЛЮИДА В СКВАЖИНЕ
}

\author{
Исмаков Рустэм Адипович',
}

ismakovrustem@gmail.com

\author{
Денисова Екатерина Всеволодовна ${ }^{2}$, \\ denisova.anrb@mail.ru
}

\author{
Черникова Марина Алексеевна, \\ chernikovamarina18@gmail.com
}

\author{
Сидоров Сергей Павлович', \\ sergeysidorov84222@gmail.com \\ 1 Уфимский Государственный нефтяной технический университет, \\ Россия, 450000, г. Уфа, ул. Космонавтов, 1. \\ 2 Уфимский Федеральный исследовательский центр Российской академии наук, \\ Россия, 450054, г. Уфа, пр. Октября, 71.
}

\begin{abstract}
Актуальность исследования состоит в том, что решением преждевременного прорыва воды или газа в горизонтальной скважине из-за неоднородности профилей притока вдоль оси горизонтального ствола, является изменение пластового давления на различных участках, а также при разработке контактных месторождений, особенно по мере истощения залежи, могут служить устройства контроля притока флюида. Различают активные Interval Control Valve (ICV) или пассивные Inflow Control Device (ICD) устройства. Устройства ICD способны выровнять приток вдоль горизонтальной скважины за счет создания дополнительного сопротивления потоку жидкости, зависящего от величины притока на данном горизонтальном участке. Недостаток современных ICD в том, что они не имеют возможности регулирования и приведения пассивных устройств в действие после установки в стволе скважины. В связи с тем, что имеются риски связанные с неопределенностью в описании свойств пласта, которые присутствуют на всех стадиях разработки месторождения недостаток ICD оказывается существенным. Системы ICV приводятся в действие дистанционно с поверхности скважины, но не способны определять характер поступающего флюида (нефть, газ, вода) в скважину и принимать решение в автоматическом режиме.

Цель: разработка новой конструктивной схемы устройства контроля притоком с возможностью непрерывного мониторинга характера поступающей жидкости, и программного обеспечения для управления клапаном с устья скважины.

объекты: горизонтальная скважина и устройство контроля притоком флюида.

Методы: имитационное моделирование Simulink, нейронные сети, матричные методы, методы линеаризации нелинейных уравнений

Результаты. Предложена новая конструктивная схема устройства контроля притока в горизонтальной скважине, позволяющая непрерывно оценивать характер поступающего флюида. Данная конструкция позволяет в автоматическом режиме регулировать положение исполнительного механизма по данным измерительных приборов. Дано математическое описание работы клапана. Разработана модель клапана в среде моделирования Simulink, с использованием матричного подхода и нейронных сетей, для построения качественной зависимости положения клапана от значения создаваемого перепада давления. Приведены результаты работы блока нейронной сети и конечный результат моделирования.
\end{abstract}

\section{Ключевые слова:}

Устройство контроля притока, горизонтальная скважина, нейронные сети, матричный подход, моделирование.

\section{Введение}

Вопрос обеспечения наиболее качественной выработки запасов нефти, на фоне истощения легко извлекаемых, дает стимул для развития технологий, позволяющих увеличить срок рентабельной разработки местрождения. Чтобы увеличить коэффициент извлечения нефти, дебит, а также площадь контакта пласта со скважиной, нефтегазовые компании активно реализуют разработку горизонтальных скважин (ГС). ГС позволяют производить разработку месторождений с риском раннего прорыва подошвенной воды или газовой шапки [1]. Но ГС не решают всех проблем, преждевременный прорыв воды или газа происходит вследствие неоднородности профилей притока вдоль всей длины ГС, перепада пластового давления в стволе сква- жины, эффекта heel-toe, который приводит к различию в удельной скорости потока между пяткой и носком скважины, особенно в однородных резервуарах $[2,3]$. Решением указанных проблем может служить устройство контроля притока (УКП). Различают пассивные Inflow Control Device (ICD) и активные Inflow Control Valve (ICV) устройства. Hедостаток современных $I C D$ в том, что они не имеют возможности регулирования и приведения пассивных устройств в действие после установки в стволе скважины. Возможность регулирования притока по длине скважины обусловлена созданием дополнительного гидравлического сопротивления, в следствии известных физических законов, регулирующих поток жидкости из резервуара через $I C D$ в эксплуатационную скважину. Различные кон- 


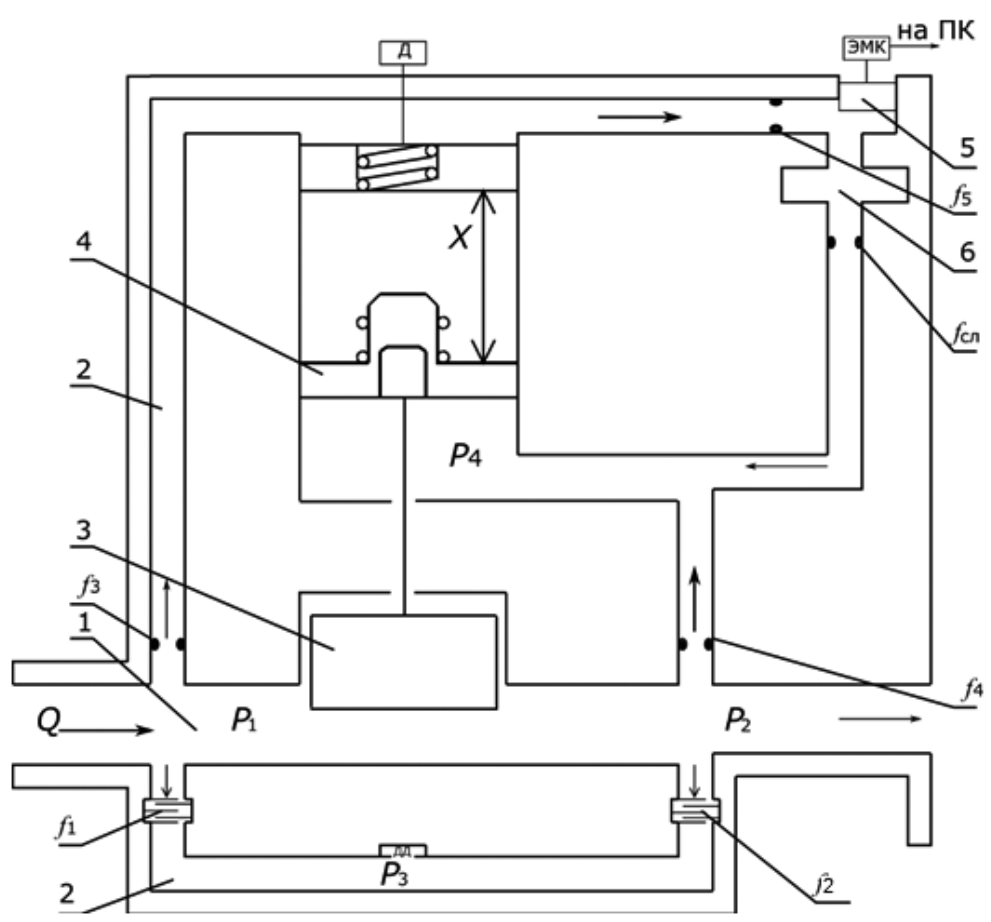

Рис. 1. Схема клапана контроля притока: 1 - основной канал, 2 - вторичный канал, 3 - исполнительный механизл, 4 - поршень, $f_{1}$, $f_{2}$ - дроссельный пакет, $f_{3} . . f_{5}, f_{\text {сл }}$ жиклер, 5 - заслонка, 6 - буферная емкость, ДД - датчик давления, Д - контроль перемещенuя

Fig. 1. Diagram of the flow control valve: 1 is the main channel, 2 is the secondary channel, 3 is the actuator, 4 is the piston, $f_{1}, f_{2}$ is the droplet pack, $f_{3} \ldots f_{5}, f_{\text {сл }}$ is the jet, 5 is the damper, 6 is the buffer capacity, ДД is the pressure sensor, $Д$ is the movement control

структивные решения устройств на данный момент включают: отверстия, сопла, трубки, спиральные и лабиринтные каналы. ICV управляется дистанционно (с поверхности) с помощью гидравлической, электрической или электрогидравлической системы управления [4], но не способны определять характер поступающего флюида (нефть, газ, вода) в скважину и принимать решение в автоматическом режиме, поэтому принято решение о создании собственной структуры УКП. Подробное описание существующих устройств контроля притоком флюида дано в литературе [5-12].

\section{Постановка задачи}

Разработка новой конструктивной схемы УКП с возможностью непрерывного мониторинга характера поступающей жидкости; составление математического описания работы устройства, имитационное моделирование системы автоматического управления клапаном.

\section{Описание конструкции и работы клапана}

Для решения поставленных задач при разработке клапана необходимо создать две области: область определения характера протекающей жидкости (нефть, вода, газ) и область непосредственного управления клапаном. На рис. 1 представлена схема клапана контроля притока, который включает основной канал - 1 и вторичный канал - 2 клапана. Во вторичном канале установлены ограничители потока в виде дроссельных паке- тов $f_{1}, f_{2}$, и жиклеров $f_{3}, f_{4}, f_{5}, f_{\text {сл }}$, ДД - датчик перепада давления. Основной канал содержит исполнительный механизм - 3 , приводимый в действие поршнем -4 , положение которого определяется с помощью датчика перемещения Д. Вторичный канал - 2 ограничен заслонкой - 5 и электромагнитным клапаном (ЭМК), приводимым в действие с поверхности скважины. Излишки, вытесненные в области заслонки, попадают в буферную емкость $6[13,14]$.

Электрогидравлический клапан контроля притока флюида работает следующим образом. Поток жидкости $Q$ проходит через основной канал - 1 , попадая из продуктивного пласта в эксплуатационную трубу. Во вторичном канале - 2 при прохождении жидкости через дроссельные пакеты $f_{1}$, $f_{2}$ происходит падение давления, которое можно настраивать, подбирая количество пластин - 1 в пакете и диаметр отверстия - 2 (рис. 2).

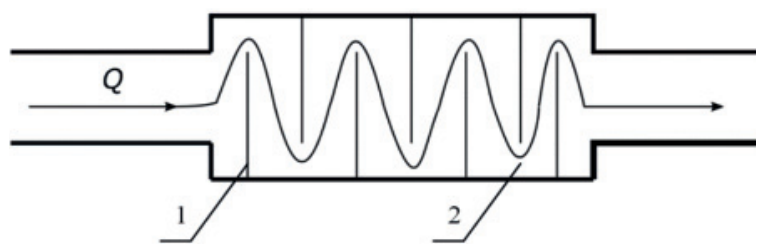

Рис. 2. Принципиальная схема дроссельного пакета: 1 - пластина, 2 - отверстие для потока

Fig. 2. Schematic diagram of throttle package: 1 is the plate, 2 is the flow hole 
Падение давления зависит от плотности, температуры и скорости поступающего флюида. Расход жидкости $Q_{1}$ при прохождении через ограничитель потока $f_{1}$ можно определить по формуле (1):

$$
Q_{1}=a \mu f_{1} \sqrt{P_{1}-P_{3}},
$$

где $a=\sqrt{\frac{2 g}{\rho}} ; g-$ ускорение свободного падения, $\mathrm{M} / \mathrm{c}^{2} ; \rho-$ плотность или удельный вес потока жидкости, кг $/ \mathrm{M}^{3} ; \mu$ - коэффициент истечения; $f_{1}$ - площадь ограничителя потока, $\mathrm{m}^{2} ; P_{1}$ - давление на входе, Па; $P_{3}$ - давление после ограничителя потока $f_{1}$, Па.

Таким образом, зная перепад давления и температуру протекающей жидкости, можно определить характер протекающего флюида в скважине при заданном расходе флюида.

Теперь составим уравнения для описания работы клапана. В уравнении (2) представлены силы, действующие на поршень:

$$
m_{\Sigma} \ddot{x}+C_{\text {пр }} x+R_{\text {пр }}=F_{\text {п }}\left(\Delta P_{5}-\Delta P_{4}\right),
$$

где $m_{\Sigma}$ - суммарная масса исполнительного механизма и поршня, кг; $x$ - перемещение поршня, м; $C_{\text {пр }}$ - коэффициент жесткости пружины, $\mathrm{H} / \mathrm{m}^{2} ; R_{\text {пр }}-$ сила предварительной затяжки на поршне, $\mathrm{H} ; F_{\text {п }}$ площадь под поршнем, $\mathrm{m}^{2} ; P_{5}$ - давление над поршнем, Па; $P_{4}$ - давление под поршнем, Па.

Проведем линеаризацию уравнения относительно малого перемещения поршня Х. Уравнение (2) [15] запишем в виде:

$$
m_{\Sigma} \Delta \ddot{x}+C_{\text {пр }} \Delta x=F_{\text {пा }}\left(\Delta P_{5}-\Delta P_{4}\right),
$$

Запишем уравнение расхода в линеаризованном виде:

$$
\Delta Q_{1}=\alpha_{1}\left(\Delta P_{1}-\Delta P_{3}\right),
$$

где $\alpha_{1}=\frac{a \mu f_{1}}{2 \sqrt{P_{1}-P_{3}}}-$ есть коэффициент линеаризации.

Расход жидкости при прохождении других ограничителей потока аналогичен (4).

$$
\begin{gathered}
\Delta Q_{3}=\alpha_{3}\left(\Delta P_{1}-\Delta P_{5}\right)=\alpha_{3} \Delta P_{5}+F_{\text {п }} \Delta \dot{x}, \\
\Delta Q_{4}=\alpha_{4}\left(\Delta P_{2}-\Delta P_{4}\right), \\
\Delta Q_{4}+\Delta Q_{\text {сл }}=F_{\text {п }} \Delta \dot{x} .
\end{gathered}
$$

Если сделать допущение, что $P_{\text {слл }} \approx P_{4}$, то

$$
\Delta Q_{\text {сл }}=\alpha_{\text {сл }} \Delta P_{4} .
$$

Решая уравнения (6) и (7) с учетом допущения (8),

$$
\alpha_{4} \Delta P_{2}+\left(\alpha_{\text {сл }}-\alpha_{4}\right) \Delta P_{4}=F_{4} \Delta \dot{x} .
$$

Поскольку можно считать $\Delta P_{2} \approx 0$ в уравнении (9), находим

$$
\Delta P_{4}=\frac{F_{\text {п }}}{\alpha_{\text {сл }}-\alpha_{4}} \Delta \dot{x} .
$$

Из уравнения (5) находим $\Delta P_{5}$, считая $\Delta P_{1} \approx 0$

$$
\Delta P_{5}=\frac{F_{\text {I }}}{\alpha_{32}-\alpha_{31}}(-\Delta \dot{x}) .
$$

Полученные зависимости (10), (11) подставляем в уравнение (3), получаем баланс сил на поршне в линеаризованном виде (уравнение Ньютона):

$$
m_{\Sigma} \Delta x+\left(\frac{F_{\text {п }}^{2}}{\alpha_{32}-\alpha_{31}}+\frac{F_{\text {п }}^{2}}{\alpha_{\text {сл }}-\alpha_{4}}\right) \Delta \dot{x}+C_{\text {пр }} \Delta x=0 .
$$

Полученное уравнение описывает динамику клапана как колебательное звено (13):

$$
W(s)=\frac{K}{T^{2} s^{2}+2 \xi T s+1},
$$

где $T=\sqrt{\frac{m}{C_{\text {i }}}}-$ время переходного процесса; $K-$ коэффициент передачи $K=\frac{\Delta x}{\Delta P_{3}}, \xi$-степень затухания.

Поскольку в уравнении (12) входят переменные величины $\left(\alpha_{i j}-\right.$ коэффициенты линеаризации), вычислять которые в каждой точке затруднительно, необходимо построить имитационную модель клапана для оценки его работоспособности.

\section{Имитационное моделирование}

Для построения математической модели системы воспользуемся нейронной сетью, которая позволяют достаточно точно отразить переходные процессы, в частности методом матричного представления динамических характеристик (ДХ). ДХ представляет зависимость трех параметров: перепад давления после ограничителя потока $f_{1}$, плотности жидкости и температуры. Суть метода заключается в фиксации параметров ДХ в виде матриц, как представлено в работе [16]. Модель работы клапана в Simulink с использованием нейронной сети на основе матричного подхода представлена на рис. 3. На вход модели поступает два значения параметров: перепад давления, после дроссельного пакета $f_{1}$, температура. С помощью блока (step) генерируется ступенчатый сигнал, посредством блоков одномерной таблицы $(\Delta P)$ и $\left(T,{ }^{\circ} \mathrm{C}\right)$ формируются значения входных параметров. Нейронная сеть (Neural Network) обучена для поиска входных значений, соответствующих и отличающихся от матричных, что позволяет оценивать все полученные результаты измерений. Более подробно об обучении сети изложено в работе $[17,18]$.

На рис. 4 представлен результат аппроксимации исходных данных нейронной сетью, где «॰» показаны расчетные данные, «0»- выход нейронной сети.

На выходе формируется значение плотности, которое соответствует входным данным, найденным в матрице по перепаду давления и изменению температуры. Значение плотности поступает на блок условного оператора (Uslovie), совместно с подсистемой (if Constant), которые принимают решение о положение клапана $0 ; 0,5 ; 1$ (процент открытия). Используя нейронную сеть, а также передаточную функцию $W$ $(s)$, полученную известными методами синтеза, рассчитывается передаточная функция регулятора [19]. С помощью блоков передаточной функции (regulator) 

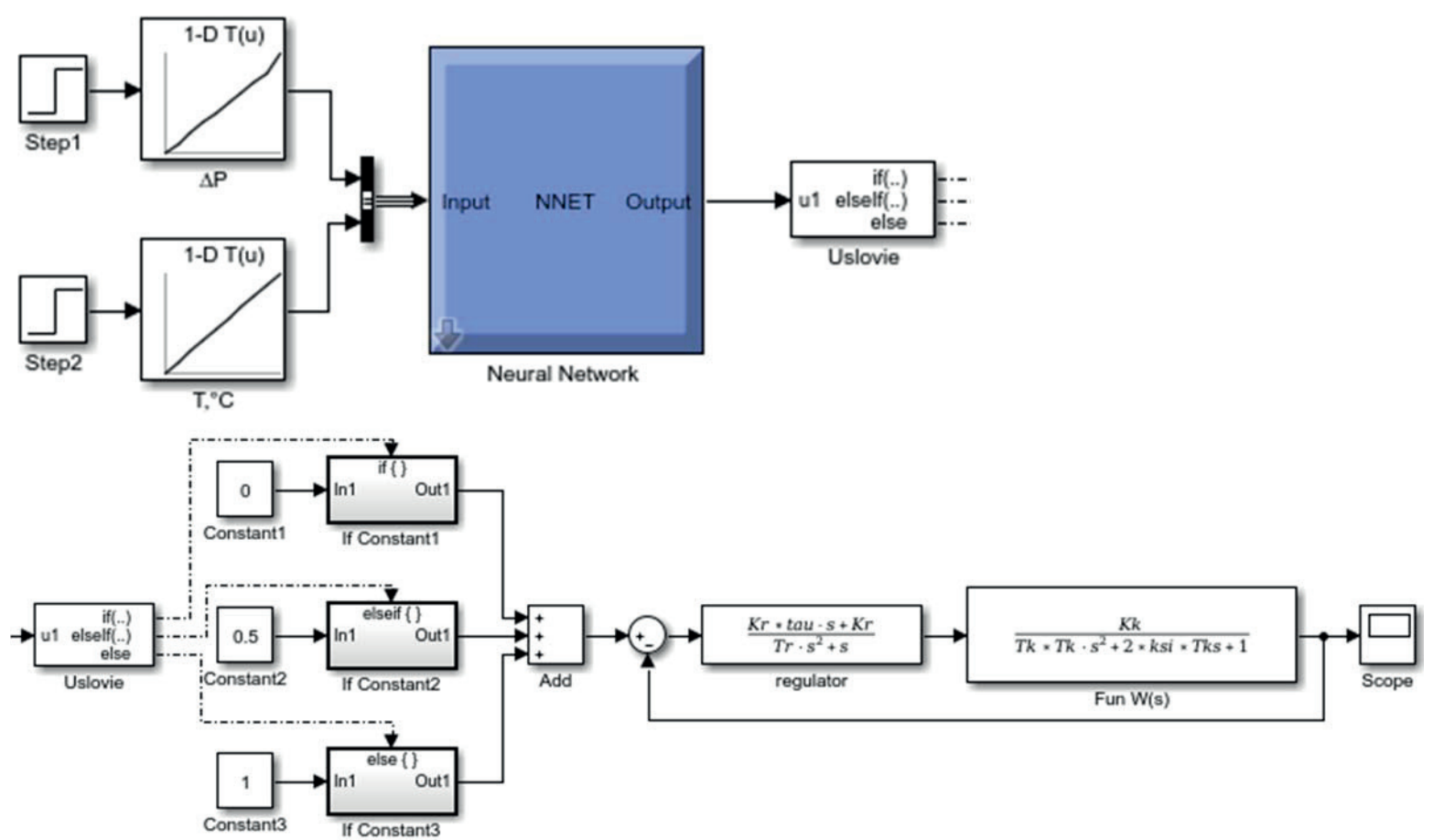

Puc. 3. Модель клапана притока флюида в Simulink

Fig. 3. Fluid flow valve model in Simulink

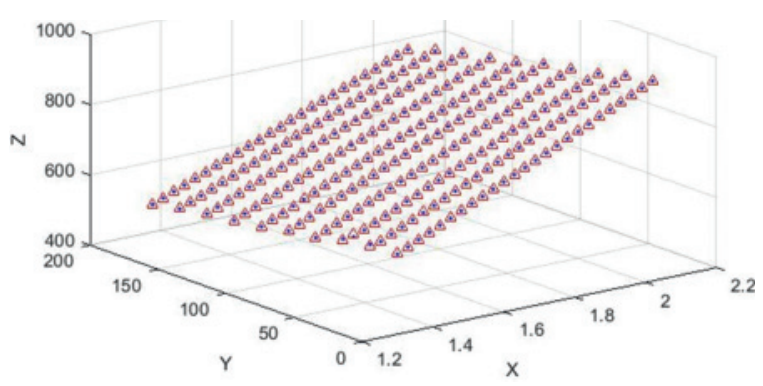

Puс. 4. Результат аппроксимации НC

Fig. 4. Result of neural network approximation и $(F u n W(s))$ задаем передаточную функцию заслонки и клапана в виде отношения полиномов: далее проверяем качество полученных переходных процессов. На рис. 5 показан переходный процесс в системе управления клапаном притока флюида.

В результате моделирования переходный процесс установился за время $t=350 \mathrm{c}$.

\section{Выводы}

1. Описана новая конструктивная схема устройства контроля притока в горизонтальной скважине, позволяющая непрерывно оценивать ха-

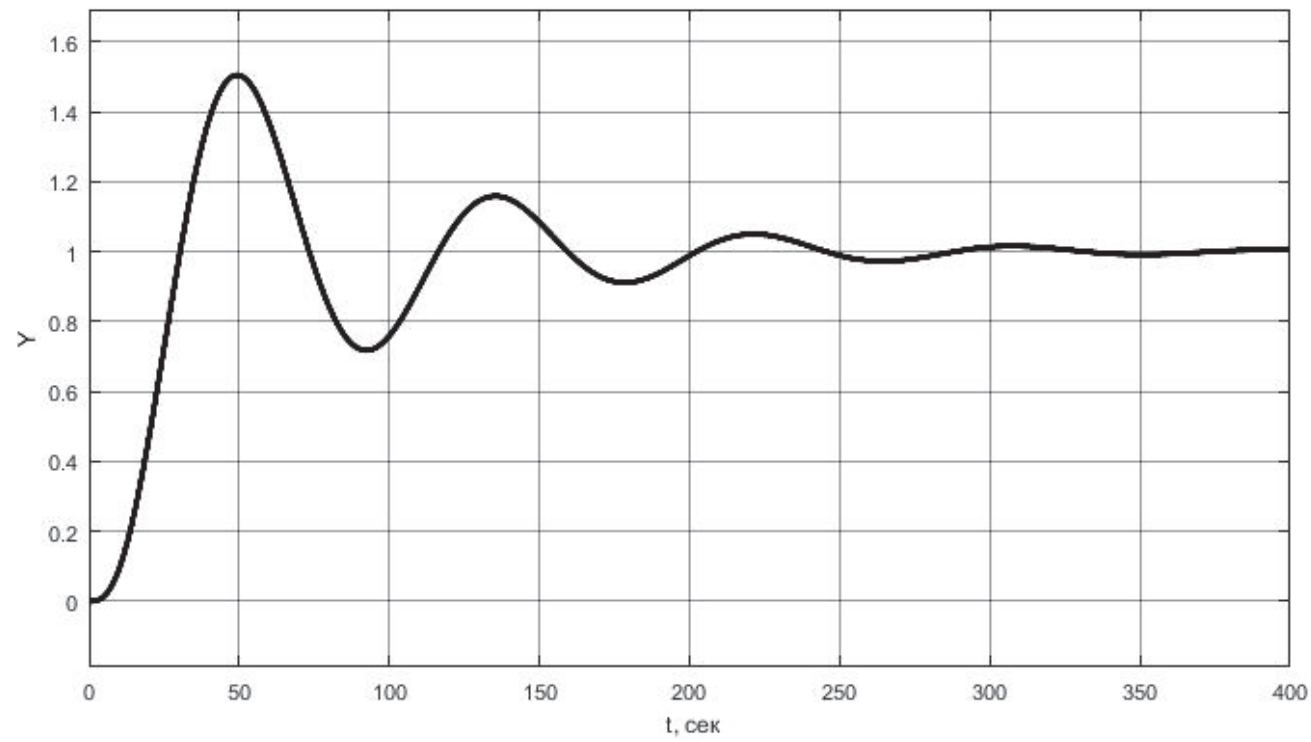

Рис. 5. Переходный процесс в системе управления клапаном притока флюида

Fig. 5. Transient in the fluid flow valve control system 
рактер поступающего флюида. Данная конструкция позволяет в автоматическом режиме регулировать положение исполнительного механизма по данным измерительных приборов.

2. Дано математическое описание работы клапана.

3. Разработана модель клапана в среде моделирования Simulink, с использованием матричного подхода и нейронных сетей, для построения качественной зависимости положения клапана от значения создаваемого перепада давления.

4. Приведены результаты работы блока нейронной сети и конечный результат моделирования.

\section{СПИСОК ЛИТЕРАТУРЫ}

1. Казымов Ш.П., Фариз А. Опыт и перспективы применения скважинных фильтров устройствами регулирования притока // НИПИ «Нефтегаз». - 2015. - Т. 2. - № 2. - С. 32-40.

2. Advanced wells: a comprehensive approach to the selection between passive and active Inflow control completions / F.T. Al-Khelaiwi, V.M. Birchenko, M.R. Konopczynski, D.R. Davies // International Petroleum Technology Conference. - Kuala Lumpur, Malaysia, 3-5 December, 2008. - P. 305-326.

3. Кувакина М. Выбор системы закачивания скважин для увеличения эффективности выработки контактных запасов (на примере Ван-Ёганского месторождения) // SPE-176525-RU. Доклад на Российской нефтегазовой технической конференции. - М., Россия, 2015. - С. 1-8.

4. Интеллектуальная скважина. URL: http://neft-gaz-novacii.ru/ru/component/content/article/56-2012-0425-12-57-58/674-1-r (дата обращения: 02.02.2018).

5. Completion technology on troll-innovation and simplicity / A. Haaland, G. Rundgren, Ø. Johannessen, Norsk Hydro // OTC 17113. Offshore technology conference. - Houston, Texas, 2-5 May 2005. - P. 1-6.

6. Henriksen K.H., Gule E.I., Augustine J. Case study: the application of inflow control devices in the troll oil field// SPE-100308 SM. Annual conference and exhibition. - Vienna, Austria, 12-15 June 2006. - P. 1-5.

7. Intelligent well technology in mature assets, paper / N. Akram, S. Hicking, P. Blythe, P. Kavanagh, P. Reijnen // SPE-71822SM. Offshore Europe conference. - Aberdeen, UK, 4-7 September 2001. - P. 1-6.

8. Improving oil production using smart fields technology in the SF30 satellite oil development offshore Malaysia / P.M. Bogaert, W. Yang, H.C. Meijers, J.C.M. van Donger, M. Konopozynski // OTC-16162-MS. Offshore technology conference. - Houston, Texas, USA, 3-6 May 2004. - P. 1-7.

9. ICD screen technology used to optimize waterflooding in injector well / A.G. Raffn, S. Hundsnes, S. Kvernstuen, T. Moen // SPE-
5. Полученные переходные процессы доказывают работоспособность системы управления клапаном притока флюида, что обеспечивает повышение качества добываемого продукта за счет своевременного отсекания поступления воды или газа [20].

\section{Заключение}

В дальнейшем планируется рассмотреть совокупность работы нескольких устройств (до шести) одновременно на одной скважине, что также повысит качество добываемого продукта.

106018-MS. Production and operations symposium. - Oklahoma City, Oklahoma, USA, 31 March - 3 April 2007. - P. 1-8.

10. Технологии регулирования притока. URL: http://www.alfahorizont.ru/index_rus/products_ru/products_filters_ru/products catalog ru 6. html (дата обрращения: 11.03.2018).

11. AI-Khelaiwi F.T., Davies D.R. Inflow control devices: application and value quantification of a developing technology // SPE108700-MS. International Oil Conference and Exhibition in Mexico. - Veracruz, Mexico, 27-30 June 2007. - P. 1-13.

12. Flow control device: Patent 20170234106A1 USA. Fil. 05.06.2017; Publ. 17.08.2017.

13. Насибуллаев И.Ш., Насибуллаева Э.Ш., Денисова Е.В. Динамика течения жидкости в технических системах с жиклерами // Известия Уфимского научного центра Российской академии наук. - 2015. - Т. 4. - С. 20-25.

14. Гусев А.А. Гидравлика. Теория и практика. 2-е изд. испр. и доп. - М.: Изд-во «Юрайт», 2014. - 285 с.

15. Бесекерский В.А., Е.В. Попова Теория систем автоматического регулирования. 3-е изд., исправ. - М.: Наука, 1975. - 768 с.

16. Денисова Е.В., Черникова М.А. Новый подход к проектированию систем автоматического управления силовыми установками беспилотных летательных аппаратов // Международный журнал прикладных и фундаментальных исследований. 2016. - № 12 (ч. 1). - С. 13-18.

17. Денисова Е.В., Мигранов А.Б., Черникова М.А. Матричный подход к моделированию газотурбинного двигателя с использованием нейронных сетей // Современные наукоемкие технологии. - 2018. - № 3. - С. 35-40.

18. Хайкин С. Нейронные сети. 2-е изд. - М.: Вильямс, 2006. $768 \mathrm{c}$

19. Черных И.В. Simulink: Инструмент моделирования динамических систем. - СПб: ДМК Пресс, 2008. - 400 с.

20. Электрогидравлический клапан притока: пат. Рос. Федерация, № 181704, заявл. 12.04.2018; опубл. 26.07.2018. Бюл. № 21. -5 с.

Поступила: 12.11 .2018 г.

\section{Информация об авторах}

Исмаков P.A., доктор технических наук, профессор, действительный член (академик) РАЕН, Почетный нефтяник, Почетный работник высшего профессионального образования Российской Федерации, проректор по научной и инновационной работе Уфимского Государственного нефтяного технического университета.

Денисова E.B., кандидат технических наук, ведущий научный сотрудник, доцент Института механики им. Р.Р. Мавлютова Уфимского Федерального исследовательского центра Российской академии наук.

Черникова М.А., младший научный сотрудник Института механики им. Р.Р. Мавлютова Уфимского Федерального исследовательского центра Российской академии наук.

Сидоров С.П., магистр кафедры автоматизации технологии производственных процессов Уфимского Государственного нефтяного технического университета. 
UDC 681.5

\title{
SYSTEM OF DEVICE FOR CONTROLLING FLUID INJECTION IN A WELL
}

Rustem A. Ismakov',

ismakovrustem@gmail.com

\section{Ekaterina V. Denisova,}

denisova.anrb@mail.ru

Marina A. Chernikova, chernikovamarina18@gmail.com

\author{
Sergey P. Sidorov', \\ sergeysidorov84222@gmail.com \\ ${ }^{1}$ Ufa State Petroleum Technological University, \\ 1, Kosmonavtov street, Ufa, 450000, Russia. \\ 2 Ufa Federal Research Centre of the Russian Academy of Sciences, \\ 71, Oktyabrya avenue, Ufa, 450054, Russia.
}

The relevance of the research is in the fact that fluid flow control devices can serve as a practical solution to premature breakthrough of water or gas in a horizontal well due to heterogeneity of inflow profile along the axis of the horizontal trunk, changes in reservoir pressure at various sites, as well as development of contact fields, especially as the deposits are depleted, serve as fluid control devices. There are active Interval Control Valve (ICV) or passive Inflow Control Device (ICD). ICD devices are able to equalize the inflow along the horizontal well, creating additional resistance to fluid flow, depending on the amount of inflow in this horizontal section. The drawback of modern ICDs is that they do not have the ability to regulate and activate ICD after installation in the wellbore. This creates certain risks associated with the uncertainty in description of reservoir properties that are present at all stages of field development. ICV systems are operated remotely from the surface of the well, but are not able to determine the nature of the incoming fluid (oil, gas, water) into the well and make a decision in automatic mode.

Objects of the research are a horizontal wells and a fluid flow control device.

Methods: simulation Simulink, neural networks, matrix methods, methods of linearization of nonlinear equations.

The main aim of the research is to development of a new design scheme of the inflow control device, with the possibility of continuous monitoring of the nature of the incoming liquid, and software for controlling the valve with the wellhead.

Results. The authors have proposed a new design scheme of inflow control device in a horizontal well, which helps continuous assessment the nature of the incoming fluid. This design allows you automatically adjust the position of the actuator mechanism, as reported by measuring instruments. The mathematical description of the valve operation is given. The model of the valve was developed in Simulink simulation environment, using the matrix approach and neural networks, to build a qualitative dependence of the valve position on pressure drop value. The results of the neural network block operation and the final result of modeling are presented.

\section{Key words:}

Inflow control device, horizontal well, neural networks, matrix approach, modeling.

\section{REFERENCES}

1. Kazymov Sh.P., Fariz A. Experience and prospects of using downhole filters with inflow control devices. Oil Gas Scientific Research Project Institute, 2015. vol. 2, no. 2, pp. 32-40. In Rus.

2. Al-Khelaiwi F.T., Birchenko V.M., Konopczynski M.R. Davies D.R. Advanced Wells: a comprehensive approach to the selection between passive and active inflow control. Completions Inter national Petroleum Technology Conference. Kuala Lumpur, Malaysia, 3-5 December, 2008. pp. 305-326.

3. Kuvakina M. Vybor sistemy zakachivaniya skvazhin dlya uvelicheniya effektivnosti vyrabotki kontaktnykh zapasov (na primere Van-Eganskogo mestorozhdeniya) [Selection of well injection system to increase the efficiency of development of contact reserves (on the example of Van-Eganskoe field)]. SPE-176525$R U$. Rossiyskaya neftegazovaya tekhnicheskaya konferentsiya [SPE-176525-RU. Russian oil and gas engineering conference]. Moscow, Russia, 26-28 October 2015. pp. 1-8.

4. Intellektualnaya skvazhina [Intelligent well]. Available at: http://neft-gaz-novacii.ru/ru/component/content/article/56-2012-04-25-12-57-58/674-l-r (accessed 2 February 2018).
5. Haaland A., Rundgren G., Johannessen Ø., Norsk Hydro. Completion technology on troll-innovation and simplicity. OTC 17113. Offshore technology conference. Houston, Texas, 2-5 May, 2005. pp. 1-6.

6. Henriksen K.H., Gule E.I., Augustine J. Case study: the application of inflow control devices in the troll oil field. SPE-100308SM. Annual Conference and Exhibition. Vienna, Austria, 12-15 June, 2006. pp. 1-5.

7. Akram N., Hicking S., Blythe P., Kavanagh P., Reijnen P. Intelligent well technology in mature assets. SPE-71822-SM. Offshore Europe conference. Aberdeen, UK, 4-7 September, 2001. pp. 1-6.

8. Bogaert P.M., Yang W., Meijers H.C., Van Donger J.C.M., Konopozynski M. Improving oil production using smart fields technology in the SF30 satellite oil development offshore Malaysia paper. OTC-16162-MS. Offshore Technology Conference. Houston, Texas, USA, 3-6 May, 2004. pp. 1-7.

9. Raffn A.G., Hundsnes S., Kvernstuen S., Moen T. ICD screen technology used to optimize waterflooding in injector well. SPE106018-MS. Production and operations symposium. Oklahoma City, Oklahoma, USA, 31 March - 3 April 2007. pp. 1-8. 
10. Tehnologii regulirovaniya pritoka [Inflow control technologies]. Available at: http://www.alfahorizont.ru/index_rus/products_ru/products_filters_ru/products_catalog_ru_6.html (accessed 11March 2018).

11. AI-Khelaiwi F.T., Davies D.R. Inflow control devices: application and value quantification of a developing technology. SPE108700-MS. Production and Operations Symposium. Oklahoma City, Oklahoma, USA, 31 March - 3 April 2007. pp. 1-13.

12. Mathiesen V., Werswick B., Aakre H. Flow control device. Patent USA, no. US 2017/0234106 A1, 2017.

13. Nasibullaev I.Sh, Nasibullaeva E.Sh, Denisova E.V. Dynamics of fluid flow in technical systems with jets. Proceedings of the RAS Ufa Scientific Centre, 2015, vol. 4, pp. 20-25. In Rus.

14. Gusev A.A. Gidravlika. Teoriya i praktika [Fluid mechanics. Theory and practice]. Moscow, Yurayt Publ., 2014. 285 p.

15. Besekerskiy V.A., Popova E.V. Teoriya sistem automaticheskogo regulirovaniya [Theory of automatic control systems]. Moscow, Nauka Publ., 1975. 768 p.
16. Denisova E.V. Chernikova M.A. A new approach to the design of automatic control systems for power plants of unmanned aerial vehicles. International journal of applied and fundamental research, 2016, vol. 12 (P. 1), pp. 13-18. In Rus.

17. Denisova E.V., Migranov A.B., Chernikova M.A. Matrix approach to modeling a gas turbine engine using neural networks. $M o$ dern high technologies, 2018, no. 3, pp. 35-40. In Rus.

18. Khaykin S. Neyronnye seti [Neural networks]. Moscow, Vilyams Publ., 2006. $768 \mathrm{p}$.

19. Chernykh I.V. Simulink: Instrument modelirovaniya dinamicheskikh sistem [Simulink: dynamic systems modeling tool]. St- Petersburg, DMK Press, 2008. 400 p.

20. Bakhtizin R.N., Ismakov R.A., Denisova E.V., Shakirova A.I., Sidorov S.P. Elektrogidravlicheskiy klapan pritoka [Electro-hydraulic inflow valve]. Patent RF, no. 2018113357/03 (021017), 2018.

Received: 12 November 2018.

\section{Information about the authors}

Rustem A. Ismakov, Dr. Sc., professor, full member (academician) of the Russian Academy of Natural Sciences, vice-rector for scientific and innovative work, Ufa State Petroleum Technological University.

Ekaterina $V$. Denisova, Cand. Sc., associate professor, leading researcher, Ufa Federal Research Centre of the Russian Academy of Sciences.

Marina A. Chernikova, junior researcher, Ufa Federal Research Centre of the Russian Academy of Sciences.

Sergei P. Sidorov, master, Ufa State Petroleum Technological University. 